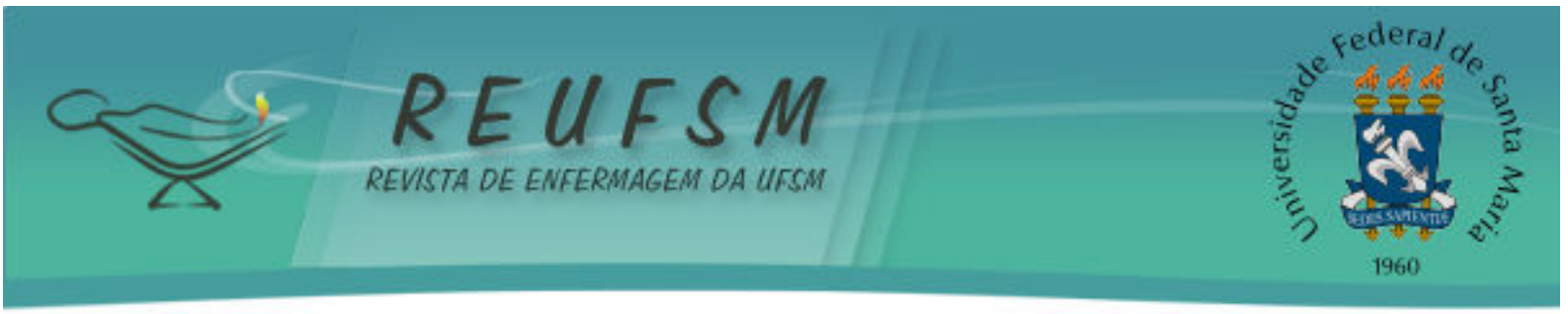

ARTIGO ORIGINAL

\title{
USO DE SUBSTÂNCIAS PSICOATIVAS POR ESTUDANTES RURAIS: DISTRIBUIÇÃO POR SEXO E IDADE
}

\section{USE OF PSYCHOACTIVE SUBSTANCES BY STUDENTS OF A RURAL SCHOOL: DISTRIBUTION BY SEX AND AGE}

\section{CONSUMO DE SUSTANCIAS PSICOACTIVAS EN UNA ESCUELA RURAL: DISTRIBUCIÓN POR SEXO Y EDAD}

Doi: $10.5902 / 2179769214144$

Ana Karla da Silva Freire 1 Bruno Passos Sampaio ${ }^{2}$ Deisiluce Miron Cavalcante ${ }^{3}$

Filipe Pitágoras Rodrigues Magalhães ${ }^{4}$ Marianna Fernandes Miranda ${ }^{5}$ Maria de Fátima Alves Aguiar Carvalho ${ }^{6}$ Mônica Cecília Pimentel de Melo ${ }^{7}$

RESUMO: Objetivo: analisar o uso na vida de álcool, tabaco e outras drogas e sua distribuição por sexo e idade em estudantes do fundamental II e médio, de uma escola pública e rural. Método: estudo quantitativo, descritivo e transversal, realizado de agosto a novembro de 2011 com 146 estudantes de Petrolina e analisado pelo programa Microsoft Excel 2007. Como critérios de elegibilidade elegeu-se ser maior de 18 anos e assinar o Termo de Consentimento Livre e Esclarecido ou, se menor, obter a assinatura do responsável. Resultados: verificou-se maior uso na vida por álcool, predominando mulheres de 16 a 18 anos; 16,44\% usaram cigarro, sem distinção do sexo; e 3,42\% usaram drogas ilícitas, prevalecendo os homens. Conclusões: estratégias educacionais, através da escola, família ou governo, são necessárias na promoção de diálogos com os alunos, reduzindo o uso de álcool, tabaco e outras drogas entre estudantes.

Descritores: Etanol; Tabaco; Drogas ilícitas; Estudantes; Ensino Fundamental e Médio.

ABSTRACT: Objective: to analyze the lifetime use of alcohol, tobacco and other drugs, and their distribution by sex and age of students of elementary and high school, in a rural public school. Method: quantitative, descriptive and cross-sectional study, conducted from August to November of 2011 with 146 students in Petrolina and analyzed by Microsoft Excel 2007. As for the criteria of eligibility, elligible students had to be over 18 and sign the Informed Consent or, if underage, obtain the parents' signature. Results: there was greater lifetime use of alcohol, predominantly among women of 16-18 years old; $16.44 \%$ used cigarettes, without distinction of sex; and $3.42 \%$ used illicit drugs,

\footnotetext{
Enfermeira, Universidade Federal do Vale do São Francisco - UNIVASF, Petrolina, PE, Brasil. E-mail: akarlasf@hotmail.com;

${ }^{2}$ Acadêmico de Medicina, Universidade Federal do Vale do São Francisco - UNIVASF, Petrolina, PE, Brasil. E-mail: brunopsam@gmail.com;

3 Psicóloga, Universidade Federal do Vale do São Francisco - UNIVASF, Petrolina, PE, Brasil. E-mail: deisylucym@hotmail.com;

${ }^{4}$ Educador Físico, Universidade Federal do Vale do São Francisco - UNIVASF, Petrolina, PE, Brasil. E-mail: filipepitagoras@hotmail.com;

5 Médica, Universidade Federal do Vale do São Francisco - UNIVASF, Petrolina, PE, Brasil. E-mail: marianna_fernandes@yahoo.com.br;

${ }^{6}$ Enfermeira, Docente em Enfermagem da Universidade Federal do Vale do São Francisco, UNIVASF, Petrolina, PE, Brasil. Mestre em Psicologia. E-mail: maria.aguiar@univasf.edu.br;

7 Enfermeira, Docente em Enfermagem da Universidade Federal do Vale do São Francisco, UNIVASF, Petrolina, PE, Brasil. Mestre em Enfermagem. E-mail: monquinamelo@gmail.com.
} 


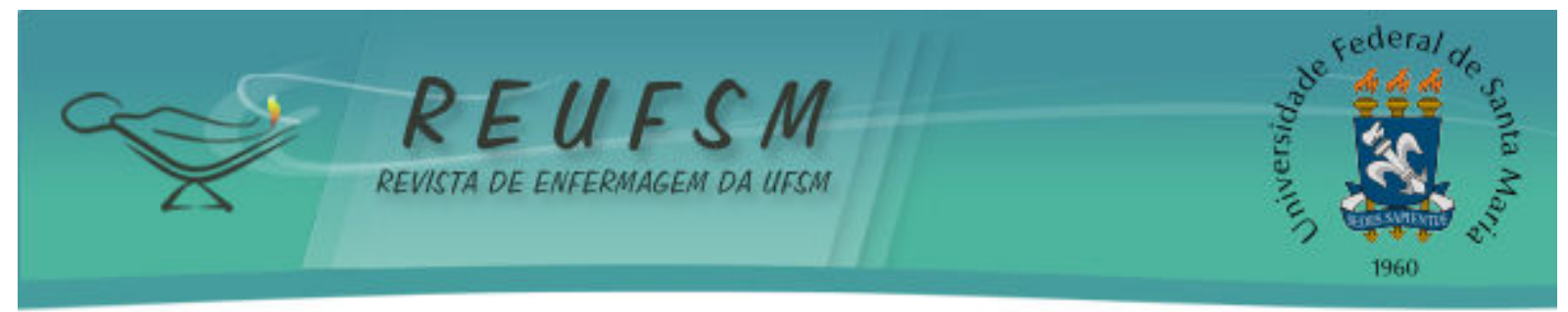

prevailing men. Conclusions: educational strategies, through school, family or government, are necessary to promote dialogues with students, reducing the use of alcohol, tobacco and other drugs among students.

Descriptors: Ethanol; Tobacco; Illicit drugs; Students; Elementary and Secondary Education.

RESUMEN: Objetivo: analizar el consumo de alcohol, tabaco y otras drogas y su distribución por sexo y edad entre estudiantes de una escuela pública rural de primaria y secundaria. Método: estudio cuantitativo, descriptivo y transversal, realizado entre agosto y noviembre de 2011 con 146 estudiantes en Petrolina (PE, Brasil) y analizados con Microsoft Excel 2007. Como criterios de elegibilidad se exigió ser mayor de 18 años y firmar un consentimiento informado o, para menores de edad, obtener la firma del responsable. Resultados: se verificó mayor consumo de alcohol, predominantemente entre mujeres de 16-18 años; $16,44 \%$ consumen cigarrillos, sin distinción de sexo; y 3,42\% consumen drogas ilícitas, prevaleciendo los hombres. Conclusiones: estrategias educativas, a través de la escuela, la familia o el gobierno, son necesarios en la promoción de diálogos con los estudiantes, para reducir el consumo de alcohol, tabaco y otras drogas entre los estudiantes.

Descriptores: Etanol; Tabaco; Drogas ilícitas; Estudiantes; Educación Primaria y Secundaria.

\section{INTRODUÇÃO}

Adolescentes são indivíduos que estão na faixa etária de 10 a 19 anos segundo a Organização Mundial de Saúde (OMS) ou entre 12 a 18 anos, segundo o Estatuto da Criança e do Adolescente (ECA). ${ }^{1-2}$ É, justamente, esse período de transformação física e comportamental que predispõe o jovem a situações nunca antes experimentadas, tornando-os mais vulneráveis ao uso de álcool e/ou outras drogas. ${ }^{3-4}$

A fase precisa ser observada com cautela, uma vez que o uso de drogas tem-se iniciado cada vez mais precocemente, intensificando-se nesse estágio. ${ }^{5}$ É um momento de vulnerabilidades e, portanto, caracterizado pela procura e por descobertas, nas quais, os indivíduos relacionam-se com grupos de mesma afinidade, e nessa participação em novos espaços, os mesmos tornam-se mais propensos ao consumo e à dependência. ${ }^{6}$ Tornou-se uma realidade muito presente também em municípios brasileiros menores, considerado um problema de ordem social e pública. ${ }^{4}$

Nesse tocante, a escola é o espaço de integração que promove desde a infância a inserção do indivíduo na coletividade, tornando-se, assim, construtora de relações sociais e permitindo que seja um espaço privilegiado no processo de prevenção antidrogas e promoção à saúde. ${ }^{7}$

Porém, apesar de o contexto escolar contribuir positivamente nesse processo, a fragilidade e a alteração do papel social exercido faz com que esses indivíduos sintam dificuldade em enfrentar as normas impostas pela sociedade vigente. ${ }^{8}$ Com isso, a realidade nos Estados, cidades e os enfrentamentos acerca da temática precisam ser levadas em consideração. ${ }^{8}$

Assim sendo, no Brasil, o estado de Pernambuco é considerado o polígono da maconha e diante do fato, a Secretaria de Defesa Social de Pernambuco tem tentado combater o aumento do uso de drogas pela faixa etária ao desenvolver o projeto "Pernambuco contra o crack". 9-10 0 estado compreende as cidades localizadas nas regiões dos Sertões e do São Francisco e são destacadas pela intensa produção e tráfico de 


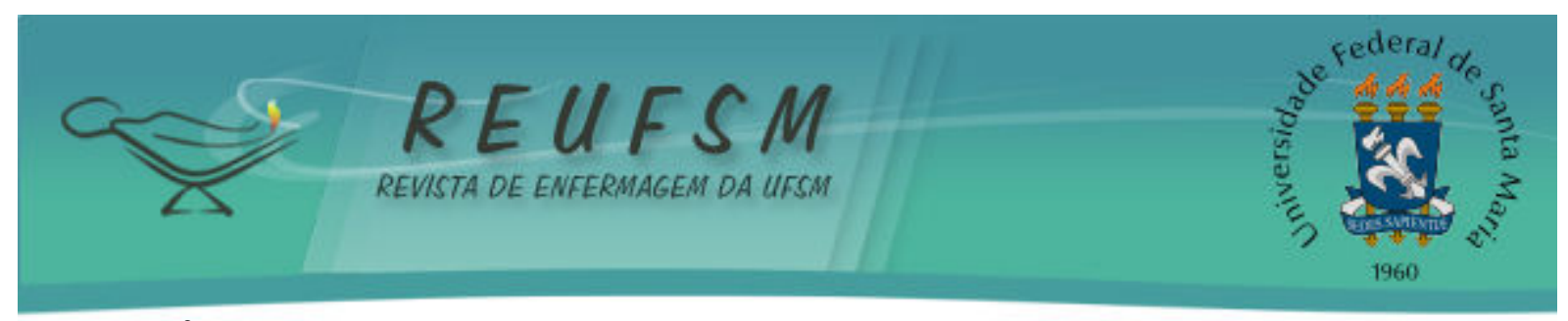

maconha. ${ }^{9}$ Uma dessas é Petrolina, a qual é lócus deste estudo e está localizada na região do submédio do Vale do São Francisco.

Estudos sobre a temática são mais prevalentes nas capitais e grandes centros urbanos e escassos em interiores do país. ${ }^{11}$ Além disso, o consumo de álcool e outras drogas tem despertado, no Brasil, uma inquietação sobre a política pública referente à definição desta. ${ }^{12}$ Assim sendo, a justificativa em desenvolver essa pesquisa entre adolescentes de uma escola rural do município de Petrolina está pautada na complexidade do assunto e no número de usuários existentes com seu respectivo impacto sobre indivíduos e sociedade. ${ }^{5-6,9,11}$

A partir disso, surgiu como questão de pesquisa: qual a distribuição por sexo e idade quanto ao uso na vida de drogas lícitas e ilícitas por estudantes de uma escola pública e rural de Petrolina-PE? Como objeto destaca-se o uso na vida de drogas lícitas e ilícitas por estudantes, sendo pertinentes programas e projetos que visem à promoção e prevenção do uso de drogas, abrangendo toda a população, e em especial adolescentes, os quais se mostram vulneráveis ao uso de substâncias psicoativas.

Pretendeu-se, assim, como objetivo, analisar o uso na vida, entendido como o consumo pelo menos uma vez na vida, de álcool, tabaco e outras drogas e sua distribuição por sexo e idade em estudantes do fundamental II e médio, de uma escola pública e rural.

\section{MÉTODO}

Trata-se de um estudo de abordagem quantitativa, descritivo e transversal, realizado com 146 alunos ( 86 do Ensino Fundamental II (de $5^{\mathrm{a}}$ a $8^{\mathrm{a}}$ séries) e 60 do Ensino Médio ( $1^{\mathrm{a}}$ a $3^{\mathrm{a}}$ séries), com faixa etária de 10 a 29 anos) de uma escola pública, na área rural do município de Petrolina (PE), distante $14 \mathrm{~km}$ do centro urbano. A população total na cidade é de 293.962 habitantes, sendo 74.747 habitantes na área rural. ${ }^{13-14}$

A amostra foi selecionada por conveniência nos turnos vespertino e noturno e a escolha dos participantes deu-se por demanda espontânea, mediante a manifestação de aceite pelos estudantes com a assinatura do aluno ou responsável (caso menor de 18 anos) do Termo de Consentimento Livre e Esclarecido - TCLE. Assim, os critérios de elegibilidade adotados foram ser maior de 18 anos e assinar o TCLE, ou se menor, obter a assinatura do responsável.

A coleta de dados foi realizada em uma escola rural de Petrolina (PE) durante o horário de aula. Realizada no período de agosto a novembro de 2011, por estudantes dos cursos de ciências farmacêuticas, educação física, enfermagem, medicina e psicologia, os quais fazem parte das ações do Programa de Educação pelo Trabalho - Saúde Mental crack/álcool e outras drogas (PET-Saúde mental).

Os alunos que confirmaram participar da pesquisa responderam a um questionário baseado no instrumento elaborado pelo Centro Brasileiro de Informações sobre Drogas Psicotrópicas (CEBRID), padronizado, autopreenchível e não identificável. ${ }^{15}$ Esse questionário continha 35 questões fechadas e de múltipla escolha sobre o uso de álcool, tabaco, drogas inalantes (lança-perfume, loló, cola, gasolina, acetona, tíner e éter) e drogas ilícitas (maconha, cocaína, crack, ecstasy, heroína e LSD).

As respostas foram digitadas em um banco de dados na Planilha Eletrônica do Microsoft Excel 2007 e a análise foi feita mediante estatística descritiva através da formulação de tabelas dinâmicas e gráficos, permitindo assim, a discussão dos resultados.

Foram respeitados os aspectos éticos e legais estabelecidos na Resolução 196/96 do Conselho Nacional de Ética e Pesquisa em Seres Humanos, sendo o instrumento e os procedimentos da pesquisa aprovados pelo Comitê de Ética da Universidade Federal do Vale do São Francisco, aprovado dia 27 de maio de 2011, sob parecer nº 0018/270512. 


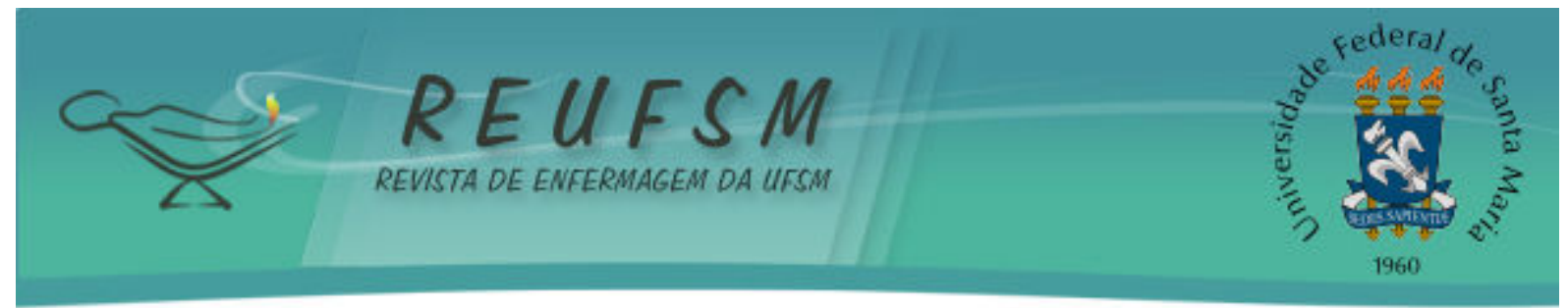

\section{RESULTADOS}

A análise dos resultados mostrou que dos 146 estudantes que compunham a amostra, $58,90 \%$ eram do sexo feminino e $40,41 \%$ do sexo masculino, distribuídos nas seguintes faixas etárias: de 10 a 14 anos (37,90\%), de 15 a 19 anos (47,49\%), de 20 a 29 anos (14,61\%). A distribuição conforme nível escolar apresentou-se da seguinte forma: $58,90 \%$ dos alunos no Ensino Fundamental (de $5^{\mathrm{a}}$ a $8^{\mathrm{a}}$ séries) e $41,10 \%$ no Ensino Médio ( $1^{\mathrm{a}}$ a $3^{\mathrm{a}}$ séries).

A porcentagem de alunos que admitiu ter feito uso na vida de álcool foi maior do que a de uso das outras substâncias, uma vez que os alunos que afirmaram ter ingerido álcool, pelo menos uma vez na vida, corresponderam a 63,01\% ( $n=92)$ da amostra (Tabela 1). Destes, $54,35 \%(n=50)$ eram mulheres e $44,57 \%(n=41)$ homens, havendo predomínio das mulheres (Tabela 2).

Tabela 1 - Uso na vida de álcool, cigarro, inalantes e outras drogas por estudantes de escola pública da área rural de Petrolina - PE, 2011

$\begin{array}{lll}\text { Uso na vida } & \text { N }\end{array}$

Álcool

Sim

Não

Cigarro

Sim

Não

Não respondeu

Drogas inalantes

Sim

Não

Não respondeu

Outras drogas

Sim

Não

Não respondeu
92

54

24

120

2

16

126

4

\section{5}

137

4

146
$63,01 \%$

$36,99 \%$

$16,44 \%$

$82,19 \%$

$1,37 \%$

$10,96 \%$

$86,30 \%$

$2,74 \%$

$3,42 \%$

$93,84 \%$

$2,74 \%$

$100,00 \%$

Tabela 2. Distribuição do uso na vida de álcool, cigarro, inalantes e outras drogas conforme sexo dos estudantes de escola pública rural. Petrolina - PE, 2011.

\begin{tabular}{lcccc}
\hline \multicolumn{1}{c}{ Sexo } & $\begin{array}{c}\text { Álcool } \\
\mathrm{n}(\%)\end{array}$ & $\begin{array}{c}\text { Cigarro } \\
\mathrm{n}(\%)\end{array}$ & $\begin{array}{c}\text { Drogas } \\
\text { inalantes } \\
\mathrm{n}(\%)\end{array}$ & $\begin{array}{c}\text { Outras drogas } \\
\text { ilícitas } \\
\mathrm{n}(\%)\end{array}$ \\
Feminino & $50(54,35 \%)$ & $12(50 \%)$ & $6(37,50 \%)$ & $2(40 \%)$ \\
Masculino & $41(44,56 \%)$ & $12(50 \%)$ & $10(62,50 \%)$ & $3(60 \%)$ \\
Não respondeu & $1(1,09 \%)$ & - & - & - \\
TOTAL & $92(100,00 \%)$ & $24(100,00 \%)$ & $16(100,00 \%)$ & $5(100,00 \%)$ \\
\hline
\end{tabular}




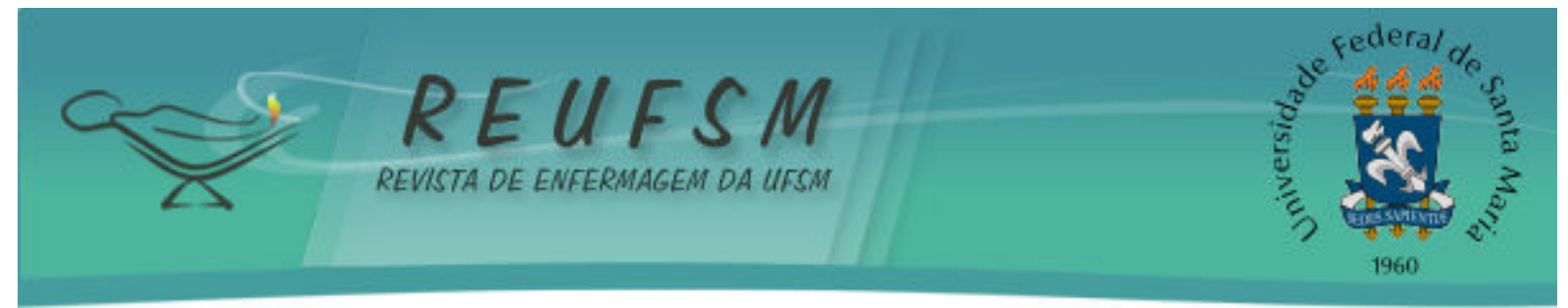

Percebe-se que o uso na vida de bebidas alcoólicas foi superior na faixa etária de 16 a 18 anos, correspondente a 33,70\% $(n=31)$ e que 8,70\% dos que já ingeriram álcool têm entre 10 e 12 anos. Importante observar também, que os indivíduos com idade acima dos 18 anos de idade representaram 30,43\% ( $n=28)$ daqueles que relataram consumo (Tabela $3)$.

Tabela 3. Distribuição do uso na vida de álcool, cigarro, inalantes e outras drogas conforme faixa etária dos estudantes de escola pública rural. Petrolina - PE. 2011

\begin{tabular}{lcccc}
\hline Faixa etária & $\begin{array}{c}\text { Álcool } \\
\mathrm{n}(\%)\end{array}$ & $\begin{array}{c}\text { Cigarro } \\
\mathrm{n}(\%)\end{array}$ & $\begin{array}{c}\text { Drogas } \\
\text { inalantes } \\
\mathrm{n}(\%)\end{array}$ & $\begin{array}{c}\text { Outras drogas } \\
\text { ilícitas } \\
\mathrm{n}(\%)\end{array}$ \\
Entre 10 e 12 anos & $8(8,70 \%)$ & - & $1(6,25 \%)$ & - \\
De 13 a 15 anos & $25(27,17 \%)$ & $4(16,67 \%)$ & $6(37,50 \%)$ & - \\
De 16 a 18 anos & $31(33,70 \%)$ & $11(45,83 \%)$ & $7(43,75 \%)$ & $4(80,00 \%)$ \\
Acima de 18 anos & $28(30,43 \%)$ & $9(37,50 \%)$ & $2(12,50 \%)$ & $1(20,00 \%)$ \\
TOTAL & $92(100,00 \%)$ & $24(100,00 \%)$ & $16(100,00 \%)$ & $5(100,00 \%)$ \\
\hline
\end{tabular}

O consumo de cigarro se configurou da seguinte forma: $16,44 \%(n=24)$ afirmaram ter feito uso pelo menos uma vez na vida de cigarro (Tabela 1), com distribuição igualitária entre os sexos (Tabela 3). Conforme a faixa etária, novamente, evidenciou-se que o grupo com maior prevalência de consumo foi aquele correspondente às idades de 16 a 18 anos $(n=11 ; 45,83 \%)$, conforme apresentado na tabela 2 .

Neste estudo, a pesquisa sobre o uso de solventes e outros inalantes, mostrou que $10,96 \%(n=16)$ do total de entrevistados tinham feito uso dessas substâncias pelo menos uma vez na vida e 2,74\% $(n=4)$ não responderam ao item (Tabela 1). 0 uso dessas substâncias foi superior entre os sujeitos do sexo masculino $(62,50 \% ; n=10)$, como mostra a tabela 2. Novamente, a faixa etária em que o consumo se mostrou mais prevalente foi entre 16 e 18 anos, sendo equivalente a 43,75\% (n=7), conforme tabela 3.

A pesquisa de uso de drogas ilícitas demonstrou que apenas $3,42 \% \quad(n=5)$ da amostra afirmaram ter feito uso ao menos uma vez na vida; 93,84\% ( $n=137)$ negaram o uso dessas substâncias e 2,74\% $(n=4)$ não responderam ao quesito (Tabela 1). Estavam entre as drogas com uso reconhecido: maconha, cocaína, crack e heroína e outras não especificadas. No caso dessas substâncias, o consumo maior $(60 \% ; n=3)$ foi por estudantes do sexo masculino (Tabela 2). Apenas indivíduos com idade de 16 ou mais anos estavam entre os que afirmaram ter feito uso de drogas ilícitas, sendo que $80 \%(n=4)$ deles tinham idades de 16 a 18 anos (Tabela 3).

\section{DISCUSSÃO}

O presente estudo mostrou resultados concordantes com diversos trabalhos realizados nos grandes centros urbanos. Porém a realidade observada abrangeu a situação do uso na vida de drogas e sua distribuição por sexo e idade entre adolescentes de uma escola pública e rural, o que torna um diferencial das pesquisas já realizadas.

No que se refere ao consumo de álcool, o uso na vida mostrou-se mais evidente, uma vez que $63,01 \%$ dos estudantes afirmaram ter ingerido álcool pelo menos uma vez, havendo certa predominância do sexo feminino entre os entrevistados. 


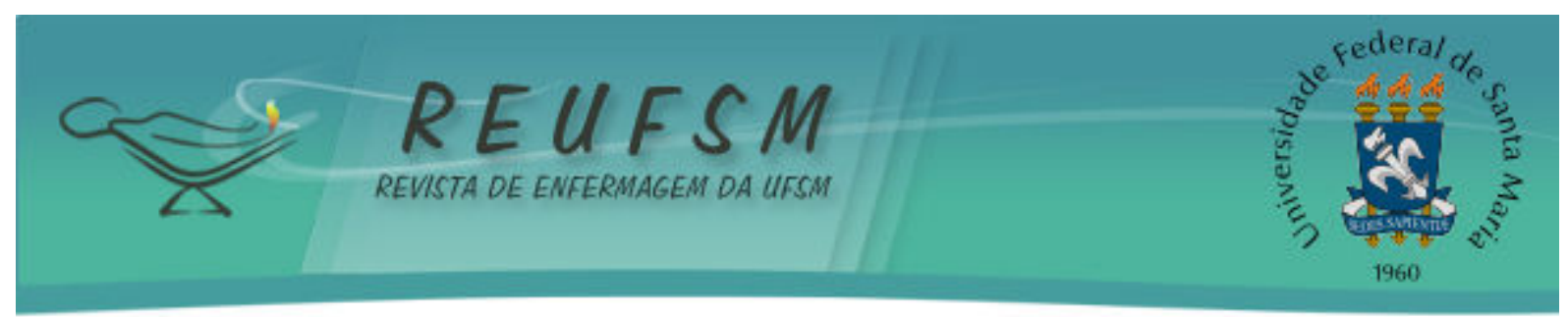

A predominância das mulheres também foi evidenciada na Pesquisa Nacional de Saúde do Escolar (PeNSE), a qual mostrou prevalência desse sexo, com uma porcentagem de $73,1 \%$ em relação a experimentação de bebida alcoólica alguma vez na vida em relação aos homens e, além disso, $71,4 \%$ de todos os estudantes pesquisados já experimentaram esse tipo de bebida em algum momento. ${ }^{16}$

Outra pesquisa elaborada pelo Centro Brasileiro de Álcool e Drogas envolvendo adolescentes de 14 a 17 anos de 143 municípios brasileiros, mostrou que $75 \%$ já tinham consumido bebida alcoólica pelo menos uma vez na vida. ${ }^{17}$ Logo, é preciso haver cautela, porque o fato de ser dispersado entre todas as pessoas que a bebida alcoólica não é uma droga, de certa forma, instiga e impulsiona o uso de álcool, em todas as faixas etárias, principalmente, no que diz respeito a adolescência. ${ }^{18}$

Observa-se ainda, que essa experimentação tem ocorrido frequentemente de maneira bastante precoce. A PeNSE revelou que em torno de três quartos dos adolescentes de 13 a 15 anos já experimentaram álcool. ${ }^{16}$ Pela pesquisa realizada em Petrolina, 8,70\% dos alunos da referida escola, que admitiram ter feito uso de bebida alcoólica, tem entre 10 e 12 anos de idade e a faixa etária com maior taxa de uso na vida $(33,70 \%)$ foi aquela de 16 a 18 anos. Semelhantemente, em estudo sobre o consumo de drogas psicoativas por adolescentes em São Paulo apresentam a faixa etária de 16 a 18 anos como os que mais fizeram uso na vida de algum tipo de bebida alcoólica. ${ }^{19}$

Dessa forma, entende-se que o álcool e o seu respectivo consumo constituem um problema de saúde pública, caracterizado pelo prejuízo na qualidade de vida que deve ser garantida pelos Municípios, Estados e União. ${ }^{18-20}$

Tal entrave é expressivo, principalmente, entre os adolescentes, uma vez que o uso exagerado proporciona, na maioria das vezes, atitudes violentas, bem como, inúmeros acidentes de trânsito, o que contribui para aumentar as mortes nessa faixa etária. ${ }^{18}$ Ademais, estudos conduzidos na Nova Zelândia e nos Estados Unidos apontam para o fato de que o indivíduo beber antes dos quatorze anos contribui como fator preditor para que usos abusivos se tornem frequentes, ocasionando em dependência durante a idade adulta..$^{21-22}$

Quanto ao tabaco, pelo último levantamento do CEBRID, foi encontrado que $16,9 \%$ dos estudantes de ensino fundamental e médio revelaram uso na vida. ${ }^{15}$ Mediante pesquisa efetuada com escolares do Distrito Federal (DF), observou-se prevalência de consumo de $10,5 \%$, sendo $9,9 \%$ no gênero masculino e $11,0 \%$ no feminino. ${ }^{23}$ Logo, existe concordância entre os dados obtidos nesta pesquisa com dados nacionais.

Em trabalho sobre o uso de álcool, tabaco e outras drogas por adolescentes escolares em um município do Sul do Brasil foram obtidos resultados bastante semelhantes ao deste. Sobre o uso pelo menos uma vez na vida, houve uma porcentagem de $60,7 \%$ no que diz respeito à bebida alcoólica. Já em relação ao tabaco, a porcentagem foi de $16,9 \%$ e sobre as outras drogas foi de $2,4 \%$ dos adolescentes. ${ }^{18}$

Os estudos, em geral, mostram que o uso de drogas lícitas - álcool e tabaco - é mais expressivo que o de drogas ilícitas entre os escolares do Brasil, o que também foi visto na presente pesquisa retratada na de acordo com a realidade rural. ${ }^{24}$ Acredita-se que esses achados sejam reincidentes, pois há uma maior disponibilidade e um acesso mais fácil a essas drogas aceitáveis e legalizadas na sociedade. ${ }^{24}$

Por outro lado, a escola é de certa forma, um fator de proteção para o uso das drogas, uma vez que o acesso às informações e melhores condições de vida contribuem significativamente para uma prevalência menor desse uso por adolescentes, em relação aos que são evadidos do sistema educacional. ${ }^{18}$

Pesquisa realizada com pais de adolescentes de uma escola pública de Curitiba trouxe que os genitores consideram o ambiente escolar como um facilitador e meio de proteção e prevenção do uso de drogas, reconhecendo a instituição como fundamental e 


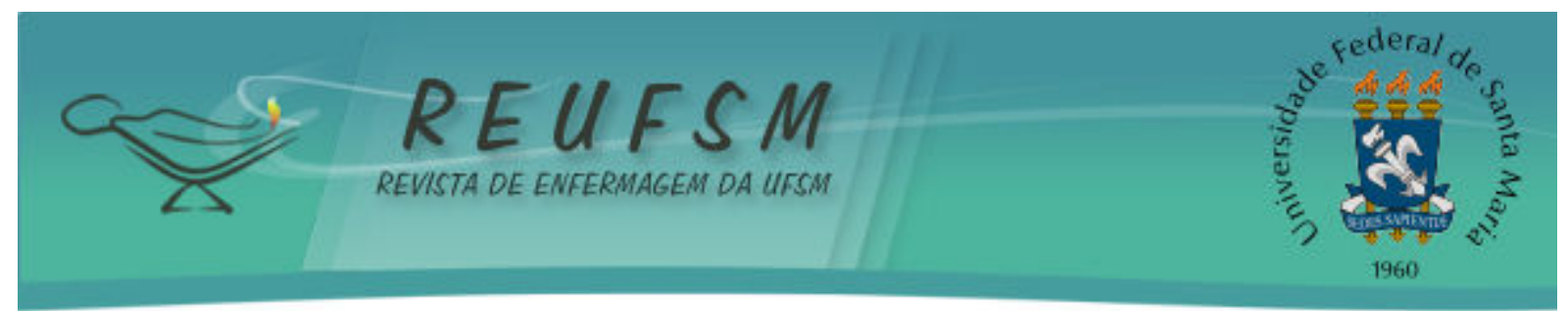

integrante na educação dos filhos. Mostraram também que é um espaço de socialização e edificação da identidade desses adolescentes fora do âmbito familiar, o que possibilita o desenvolvimento de novas habilidades, saberes e conhecimento. ${ }^{7}$

A alta prevalência no consumo de drogas entre adolescentes tem despertado pesquisadores para o estudo dos comportamentos de risco praticados por aqueles, o que é importante para a identificação dos adolescentes e do perfil desses usuários. ${ }^{25}$ Porém, ainda são escassos estudos sobre a temática na área rural.

\section{CONCLUSÃO}

Este estudo procurou analisar o uso na vida entendido como o consumo pelo menos uma vez na vida, de álcool, tabaco, e outras drogas e sua distribuição por sexo e idade em adolescentes e adultos jovens, representados por estudantes do fundamental II e médio. Com isso, percebeu-se que a maioria dos alunos destacou o uso na vida da droga lícita álcool, em comparação com as outras drogas, prevalecendo o sexo feminino, neste caso, com faixa etária mais incidente de 16 a 18 anos.

Sobre o cigarro e solventes, identificou-se que a idade de uso uma vez na vida foi a mesma do álcool, porém com distribuição igualitária entre os sexos para o primeiro e prevalência masculina no segundo.

Já as drogas ilícitas apresentaram baixo uso ao menos uma vez na vida, predominando a mesma faixa etária das outras drogas, embora o sexo masculino tenha se sobressaído em detrimento do feminino.

Nesse contexto, a pesquisa possibilitou refletir que é necessário haver uma interação entre as instituições de ensino e autoridades, sejam elas escolares, familiares ou governamentais, com o intuito de desenvolver uma aproximação e um cuidado mais efetivo, que resulte na diminuição do uso das drogas entre os estudantes.

Considera-se, então, que os objetivos da pesquisa foram alcançados, sendo, portanto, identificados, coerentemente, a realidade do uso de drogas e o perfil de adolescentes que fazem uso dessas substâncias em uma escola pública e rural. Percebeuse, assim, que os resultados, apesar de serem de uma área rural, não divergiram das pesquisas nacionais dos grandes centros urbanos, demonstrando a necessidade de mais atenção e investimentos municipais, estaduais e federais nas esferas da educação.

Além disso, mais trabalhos científicos, com ênfase no contexto rural, são necessários para se obter uma realidade mais precisa sobre o uso das drogas nesses locais. Espera-se, dessa forma, tornar visível, o cenário das drogas no ambiente escolar rural, normalmente, negligenciada pelo poder público.

Como limitação do estudo, destaca-se a impossibilidade de aplicação a todos os estudantes da escola, o que poderia resultar em uma pesquisa mais representativa.

Ademais, faz-se importante que a escola promova um espaço de escuta e de diálogo sobre a temática, permitindo a elaboração de atividades voltadas para a educação em saúde, levando em consideração a cultura local, tendo como foco o adolescente e sua família, além do planejamento de políticas destinadas à formação e à continuação de ambientes protetores e saudáveis, promovendo assim, a qualidade de vida dos alunos e de seus familiares.

\section{REFERÊNCIAS}

1. Organización Mundial de la Salud (OMS). Problemas de salud de la adolescencia [Internet]. Genebra: OMS; 1965 [acesso em 2014 out 14]. 29 p. Disponível em: 


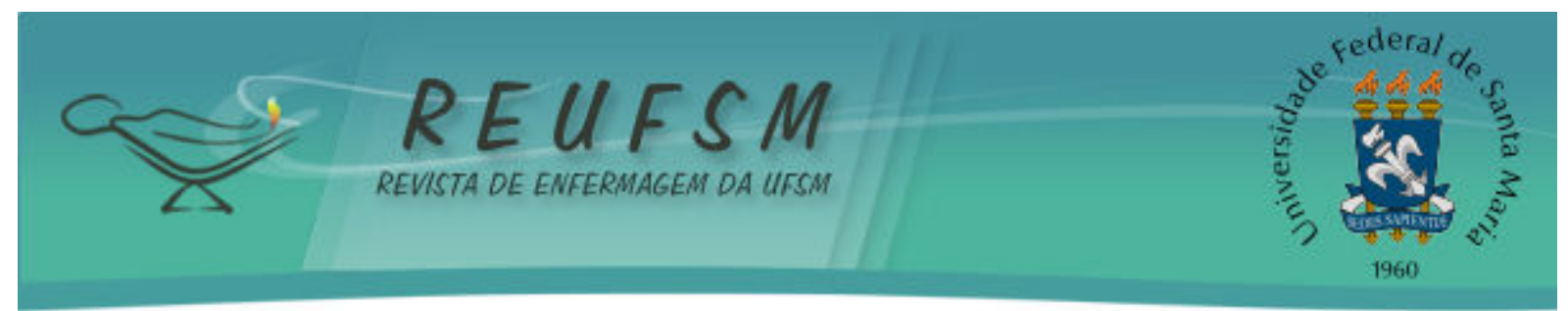

http://whqlibdoc.who.int/trs/WHO_TRS_308_spa.pdf. (OMS - Série de Informes Técnicos; 308).

2. Brasil. Estatuto da Criança e do Adolescente [Internet]. $6^{\mathrm{a}}$ ed. Brasília: Câmara dos Deputados, Coordenação de Publicações; 2008 [acesso em 2014 out 15]. Disponível em: http://bd.camara.gov.br/bd/bitstream/handle/bdcamara/14683/estatuto_crianca_adoles cente_6ed.pdf?sequence $=8$.

3. Ferreira CF, Machado RM. Equipe de Saúde da Família e o uso de drogas entre adolescentes. Cogitare Enferm [Internet]. 2013 jul/set [acesso em 2014 out 16];18(3):4829. Disponível em: http://www.revenf.bvs.br/pdf/ce/v18n3/09.pdf. DOI: $10.5380 /$ ce.v18i3.33560.

4. Garcia JJ, Pillon SC, Santos MA. Relações entre contexto familiar e uso de drogas em adolescentes de ensino médio. Rev Latinoam Enferm [Internet]. 2011 maio/jun [acesso em 2014 out 13];19(N Esp):753-61. Disponível em: http: / /www.scielo.br/scielo.php?script=sci_pdf\&pid=S0104$11692011000700013 \& \operatorname{lng}=e n \& n r m=i s o \& t \operatorname{lng}=p t$. DOI: $10.1590 /$ S0104-11692011000700013.

5. Brasil. Presidência da República. Secretaria Nacional de Políticas sobre Drogas. Andrade AG, Vieira PCA, Oliveira LG, organizadores. I Levantamento Nacional sobre o uso de álcool, tabaco e outras drogas entre universitários das 27 capitais brasileiras [Internet]. Brasília: SENAD; 2010 [acesso em 2013 mar 12]. Disponível em: http://www.obid.senad.gov.br/portais/OBID/biblioteca/documentos/Publicacoes/Univers itarios_2010/328160.pdf.

6. Monteiro CFS, Araújo TME, Sousa CMM, Martins MCC, Silva LLL. Adolescentes e o uso de drogas ilícitas: um estudo transversal. Rev Enferm UERJ [Internet]. 2012 jul/set [acesso em 2013 mar 12];20(3):344-8. Disponível em: http://www.facenf.uerj.br/v20n3/v20n3a11.pdf.

7. Brusamarello T, Maftum MA, Mazza VA, Silva AG, Silva TL, Oliveira VC. Papel da família e da escola na prevenção do uso de drogas pelo adolescente estudante. Ciênc Cuid Saúde [Internet]. 2010 out/dez [acesso em 2014 out 16];9(4):766-73. Disponível em: http://eduem.uem.br/ojs/index.php/CiencCuidSaude/article/view/13828. DOI: 10.4025/cienccuidsaude.v9i4.13828.

8. Sampaio JJC, Hermeto EMC, Carneiro C. Atividades socioculturais e abandono do uso de drogas ilícitas: percepção de adolescentes usuários em estudo de caso. Rev Baiana Saúde Pública [Internet]. 2009 jul/set [acesso em 2014 out 15];33(3):453-65. Disponível em: http://inseer.ibict.br/rbsp/index.php/rbsp/article/view/226/pdf_41.

9. Oliveira A, Zaverucha J, Rodrigues E. Polígono da maconha: contexto socioeconômico, homicídios e atuação do Ministério Público. In: Zaverucha J. Ministério Público Pernambucano e Paulista: um estudo comparativo sobre o enfrentamento do crime organizado e tráfico de drogas, Relatório final - Concursos Nacionais de Pesquisas Aplicadas em Justiça Criminal e Segurança Pública [Internet]. 2006 abr [acesso em 2013 mar 10]. Disponível em: http: //blogs.al.ce.gov.br/unipaceseguranca/files/2011/03/minist_public_pernamb_droga s-Blog.pdf.

10. MPPE e Estado traçam estratégias de combate ao crack em Petrolina. Recife. Ministério Público Estadual. Diário Oficial. Estado de Pernambuco. Ano LXXXIX. № 106. [acesso em 2013 mar 05]. Disponível em: 


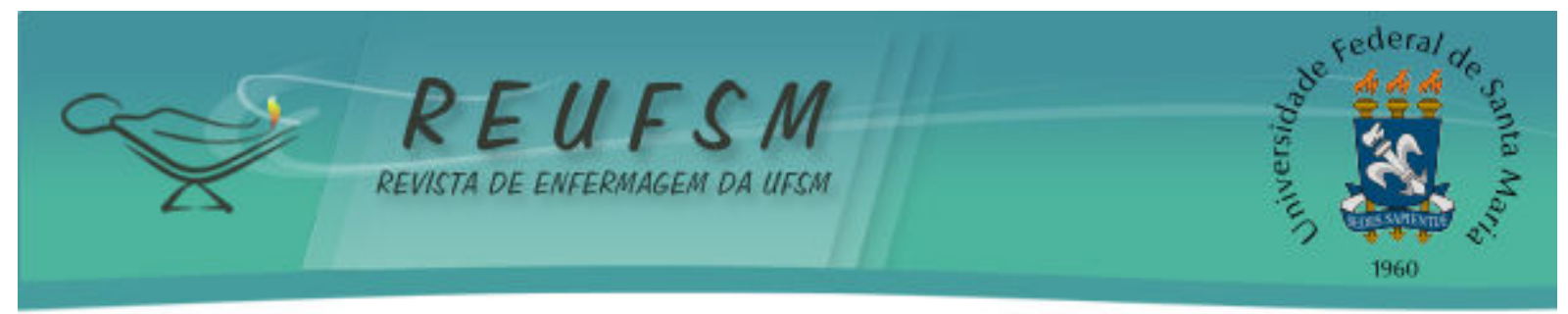

http://siteantigo.mppe.mp.br/uploads/KWux7F8dbFWDxSrx7MAujA/5I7QoHF680COg0Tfy4 U24Q/DOE_-_MPPE_07-06-2012.pdf.

11. Almeida JF, Carvalho KD, Cruz STM, Carvalho MFAA, Figueiredo, RGT. Uso de álcool entre estudantes de escolas da rede pública de ensino. Rev Enferm UFPE Online [Internet]. 2013 fev [acesso em 2013 ago 8];7(2):397-406. Disponível em: http: / / www.revista.ufpe.br/revistaenfermagem/index. php/revista/article/download/365 3/5304. DOI: 10.5205/reuol.3073-24791-1-LE.0702201310.

12. Araldi JC, Njaine K, Oliveira MC, Ghizoni AC. Representações sociais de professores sobre o uso abusivo de álcool e outras drogas na adolescência: repercussões nas ações de prevenção na escola. Interface Comum Saúde Educ [Internet]. 2012 jan/mar [acesso em 2013 out 12];16(40):135-46. Disponível em: http: / / www.scielo.br/scielo.php?script=sci_pdf\&pid=S1414$32832012000100011 \& \operatorname{lng}=e n \& n r m=i s o \& t$ lng=pt. DOI: $10.1590 /$ S1414-32832012005000002.

13. Instituto Brasileiro de Geografia e Estatística (IBGE). Censo 2014 [Internet]. Pernambuco; 2014 [acesso em 2014 out 13]. Disponível em: http: / / cidades.ibge.gov.br/painel/painel.php?codmun=261110.

14. Instituto brasileiro de Geografia e Estatística (IBGE). Censo 2010. Pernambuco; [internet] 2010 [acesso em 2014 Out 13]. Disponível em: http: //www.censo2010. ibge.gov.br/sinopse/index.php?uf=26\&dados=29.

15. VI Levantamento nacional sobre o consumo de drogas entre estudantes do ensino fundamental e médio da rede pública de ensino nas 27 capitais brasileiras 2010 [Internet]. São Paulo: CEBRID, USP; 2010. Brasília (DF): SENAD; 2010 [acesso em 2014 mar 12]. Disponível

em: http://www.obid.senad.gov.br/portais/OBID/biblioteca/documentos/Publicacoes/328890 .pdf.

16. Malta DC, Mascarenhas MDM, Porto DL, Duarte EA, Sardinha LM, Barreto SM, et al. Prevalência do consumo de álcool e drogas entre adolescentes: análise dos dados da Pesquisa Nacional de Saúde Escolar. Rev Bras Epidemiol [Internet]. 2011 set [acesso em 2012 out 15];14(1 Supl):136-46. Disponível em: http://www.scielosp.org/pdf/rbepid/v14s1/a14v14s1. DOI: 10.1590/S1415790X2011000500014.

17. Brasil. Ministério da Justiça. II Levantamento nacional sobre o uso de drogas psicotrópicas no Brasil 2005 [Internet]. Ministério da Justiça; 2007 [acesso em 2013 mar 12]. em: http://www.obid.senad.gov.br/portais/OBID/conteudo/index.php?id_conteudo=11325\&ra stro=PESQUISAS+E+ESTAT\%C3\%8DSTICAS\%2FEstat\%C3\%ADsticas/Popula\%C3\%A7\%C3\%A30+ger al+brasileira.

18. Vieira PC, Aerts DRGC, Freddo SL, Bittencourt A, Monteiro L. Uso de álcool, tabaco e outras drogas por adolescentes escolares em município do Sul do Brasil. Cad Saúde Pública [Internet]. 2008 nov [acesso em 2012 abr 23];24(11):2487-98. Disponível em: http: / /www.scielo.br/scielo.php?script=sci_pdf\&pid=S0102-

311X2008001100004\&lng=en\&nrm=iso\&tlng=pt. DOI: 10.1590/S0102-311X2008001100004.

19. Guimarães JL, Godinho PH, Cruz R, Kappann JI, Tosta Junior LA. Consumo de drogas psicoativas por adolescentes escolares de Assis, SP. Rev Saúde Pública [Internet]. 2004 fev [acesso em 2012 set 14];38(1):130-2. Disponível em: 


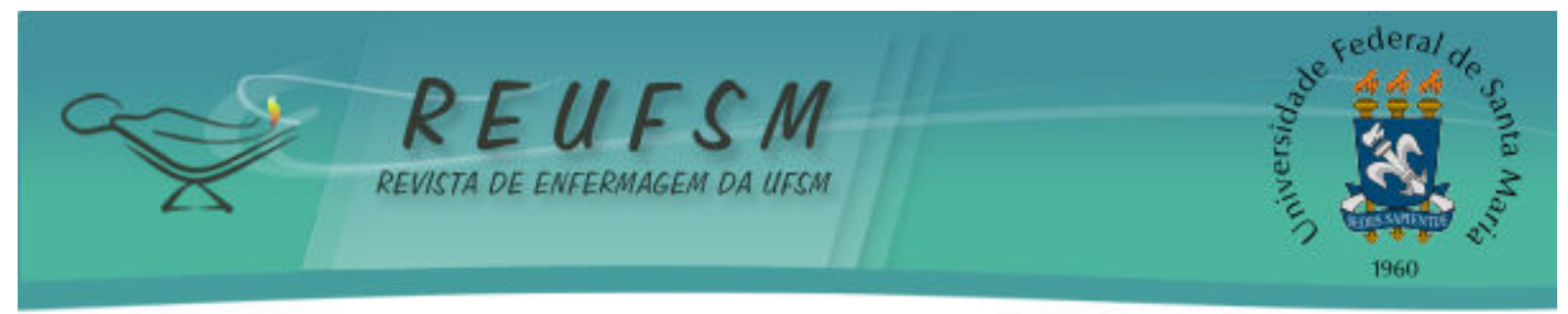

http: / / www.scielo.br/scielo.php?script=sci_pdf\&pid=S0034-

$89102004000100018 \& \operatorname{lng}=e n \& n r m=i s o \& t \operatorname{lng}=p t$. DOI: $10.1590 /$ S0034-89102004000100018.

20. Pinho A, Cecilio LPP, Neira MS, Dias DS, Severino FP, Burguetti FC. Manual básico de saúde pública: um guia prático para conhecer e garantir os seus direitos [Internet]. Penápolis: Ed. dos autores; 2012 [acesso em 2014 out 20]. Disponível em: http://www.mpsp.mp.br/portal/page/portal/Cartilhas/manual_basico_saude_publica.pdf

21. Page RM, Ihasz F, Hantiu I, Simonek J, Klarova R. Social normative perceptions of alcohol use and episodic heavy drinking among Central and Eastern European adolescents. Subst Use Misuse [Internet]. 2008 [acesso em 2013 out 28];43(3-4):361-73. Disponível em: http: //www.ncbi.nlm.nih.gov/pubmed/18365937. DOI: 10.1080/10826080701202866.

22. Terra MB, Barros HM, Stein AT, Figueira I, Athayde LD, Ott DR, et al. Predictors of relapse in 300 Brazilian alcoholic patients: a 6-month follow-up study. Subst Use Misuse [Internet]. 2008 [acesso em 2013 ago 21];43(3-4):403-11. Disponível em: http: / /www.ncbi.nlm.nih.gov/pubmed/18365940. DOI: 10.1080/10826080701202999.

23. Rodrigues MC, Viegas CAA, Gomes EL, Morais JPMG, Zakir JCO. Prevalência do tabagismo e associação com o uso de outras drogas entre escolares do Distrito Federal. J Bras Pneumol [Internet]. 2009 out [acesso em 2013 jun 16];35(10):986-91. Disponível em: http: / /www.scielo.br/scielo.php?script=sci_pdf\&pid=S1806-

$37132009001000007 \& \operatorname{lng}=e n \& n r m=i s o \& t \operatorname{lng}=$ pt. DOI: $10.1590 /$ S1806-37132009001000007.

24. Soldera M, Dalgalarrondo P, Corrêa Filho HR, Silva CAM. Uso pesado de álcool por estudantes dos ensinos fundamental e médio de escolas centrais e periféricas de Campinas (SP): prevalência e fatores associados. Rev Bras Psiquiatr [Internet]. 2004 set [acesso em 2012 mar 8];26(3):174-9. Disponível em: http: / / www.scielo.br/scielo.php?script=sci_pdf\&pid=S1516$44462004000300007 \& \operatorname{lng}=e n \& n r m=i s o \& t \operatorname{lng}=$ pt. DOI: $10.1590 /$ S1516-44462004000300007.

25. Santos MA, Pratta EMM. Adolescência e uso de drogas à luz da psicanálise: sofrimento e êxtase na passagem. Tempo Psicanál [Internet]. 2012 jun [acesso em 2013 jul 11];44(1):167-82. Disponível http: / / pepsic.bvsalud.org/scielo.php?script=sci_pdf\&pid=S0101$48382012000100010 \& l n g=p t \& n r m=i s o \& t$ lng=pt.

Data de recebimento: $26 / 05 / 2014$

Data de aceite: 05/12/2014

Contato com autor responsável: Mônica Cecília Pimentel de Melo

Endereço postal: Av. José de Sá Maniçoba S/N, bairro Centro, Petrolina - Pernambuco, Brasil. CEP 56.304-917

E-mail: monquinamelo@gmail.com 